\section{Kognitive Defizite weisen schon vor der Ersterkrankung auf eine Psychose hin}

Nicht jeder, der zur Hochrisikogruppe gehört, entwickelt tatsächlich eine Psychose. Welche weiteren messbaren Hinweise könnte man als zusätzliche Prädiktoren für eine Erkrankung verwenden?

E in großer Teil der Menschen, die zur — Hochrisikogruppe für eine Psychose gehören (aber nicht alle), entwickeln im Lauf der Zeit diese Erkrankung. Gibt es weitere Marker, die auf eine erhöhte Vulnerabilität für das Auftreten einer Psychose hinweisen?

Fusar-Poli et al. gehen in ihrer Metaanalyse der Frage nach, ob und wie stark Menschen mit einem hohen Risiko für eine spätere Psychose bereits vorher unter kognitiven Defiziten leiden.

Die Auswertung von 19 Studien mit insgesamt 1.188 Personen der Risikogruppe und 1.029 Kontrollpersonen zeigt eindeutig, dass Personen mit einem hohen Risiko vor Ausbruch einer Psychose in einer ganzen Reihe von kognitiven
Domänen (Abbildung 1) zum Teil gravierende Einschränkungen aufweisen. In der Subgruppe jener Patienten, die später tatsächlich eine Psychose entwickelten, zeigten sich dabei die deutlichsten Defizite.

Kommentar: Diese Befunde zeigen ein weiteres Mal, dass bei Patienten mit einem honen Risiko für eine Psychose bereits vor deren Beginn klinisch relevante kognitive Beeinträchtigungen vorliegen, allen voran Defizite in der Wortflüssigkeit und in den Gedächtnisdomänen. Die hier gezeigten kognitiven Defizite verweisen auf eine zerebrale Grundlage, ebenfalls existent vor Ersterkrankung.

Prof. Dr. med. Hans-Peter Volz

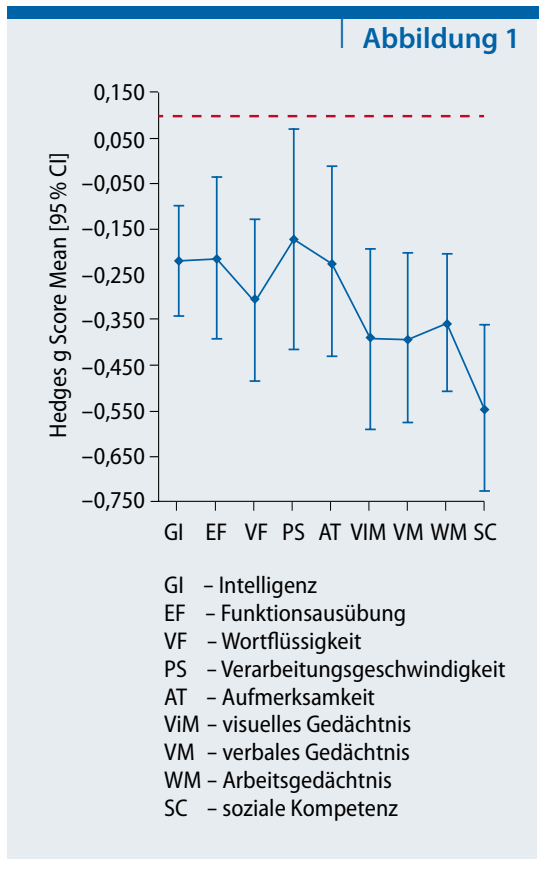

Fusar-Poli P et al. Cognitive functioning in prodromal psychosis. A meta-analysis. Arch Gen Psychiatry 2012; 69: 562-71

\title{
Dopamindysfunktion bietet - theoretisch - verschiedene Ansätze zur Therapie
}

\section{Betrachtet man die pathophysiologischen Vorgänge, die hinter einer Dopaminerhöhung stecken, erkennt man ein Potenzial für zukünftige, bisher nicht genutzte neue Therapieansätze.}

E ine Metaanalyse von 44 In-vivoStudien (insgesamt 618 Patienten mit Schizophrenie und 606 Kontrollpersonen) untersuchte, welcher Art die für die Dopaminerhöhung verantwortlichen pathophysiologischen Auffälligkeiten sind.

Diese Studien, die die dopaminerge Funktion bei schizophrenen Patienten im Vergleich zu Kontrollen untersuchten, wurden in drei Gruppen eingeteilt:

1. Studien, die die präsynaptische Funktion untersuchten (Dopamin-SyntheseAktivität, Dopaminkonzentration in der Präsynapse)

2. Studien, die die Dopaminfreisetzung untersuchten

3. Studien, die die Rezeptordichte (z.B. $\mathrm{D}_{2} / \mathrm{D}_{3}$-postsynaptische Dichte) messen.
In erster Linie zeigten die in diese Übersicht eingeschlossenen Studien, dass es robuste Befunde gibt, die auf eine erhöhte präsynaptische dopaminerge Funktion bei schizophrenen Patienten verweisen. Bei der Dopaminfreisetzung zeigten sich keine Veränderungen und die postsynaptische Rezeptordichte war nur gering erhöht.

Die Autoren stellen fest, dass die gängigen Behandlungsmethoden primär auf der Blockade der $\mathrm{D}_{2} / \mathrm{D}_{3}$-Rezeptoren beruhen, also auf Zielstrukturen, die nicht in dem Maße zur Dopaminerhöhung beitragen wie zum Beispiel die präsynaptische Dopaminsynthese, den laut der Metaanalyse robustesten Befund bei schizophrenen Patienten. Die Entwicklung zukünftiger Medikamente sollte sich unter Berücksichtigung dieser Pathophysio- logie eher an der Kontrolle der präsynaptischen Dopaminsynthese ausrichten.

Kommentar: Diese Metaanalyse zeigt meiner Ansicht nach ein erstaunliches Ergebnis insofern, als nach jahrzentelanger Forschung nur die erhöhte präsynaptische dopaminerge Funktion als robustes Ergebnis nachgewiesen werden konnte. Dies ist ernüchternd, da die vorhandenen Therapieoptionen hier nicht greifen. Allerdings ist in diesem Zusammenhang kritisch anzumerken, dass in diese Veröffentlichung nur Studien, die das Striatum untersuchten, eingeschlossen wurden. So müssen die von den Autoren gezogenen Schlussfolgerungen auf die Verhältnisse im Striatum begrenzt bleiben, sie können nicht für andere Hirnregionen generalisiert werden.

Prof. Dr. med. Hans-Peter Volz

Howes OD et al. The nature of dopamine dysfunction in schizophrenia and what this means for treatment. Meta-analysis of imaging studies. Arch Gen Psychiatry 2012; 69: 776-86 